LETTER • OPEN ACCESS

\section{Controlling adhesion using AC electric fields across fluid films}

To cite this article: Carla S Perez-Martinez et al 2021 J. Phys.: Condens. Matter 33 31LT02

View the article online for updates and enhancements.
You may also like

- Using discrete Darboux polynomials to
$\frac{\text { detect and determine preserved measures }}{\text { and integrals of rational maps }}$
E Celledoni, C Evripidou, D I McLaren et
al.
- Magnetic skyrmion-based artificial neuron
device
Sai Li, Wang Kang, Yangqi Huang et al.
- Dynamic electromagneto-mechanical
behavior of clamped-free giant
magnetostrictive/piezoelectric laminates
under AC electric fields
Kotaro Mori, Yasuhide Shindo and Fumio
Narita




\title{
Letter
}

\section{Controlling adhesion using AC electric fields across fluid films}

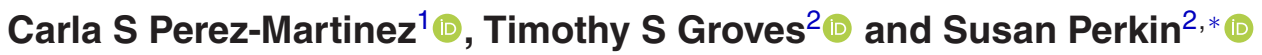 \\ ${ }^{1}$ London Centre for Nanotechnology, University College London, 17-19 Gordon Street, London WC1H \\ 0AH, United Kingdom \\ 2 Physical and Theoretical Chemistry Laboratory, University of Oxford, South Parks Road, Oxford OX1 \\ 3QZ, United Kingdom \\ E-mail: susan.perkin@chem.ox.ac.uk
}

Received 17 March 2021, revised 6 May 2021

Accepted for publication 21 May 2021

Published 15 June 2021

\begin{abstract}
We demonstrate reversible and switchable actuation using AC electric fields to bring two surfaces separated by a thin film of ionic fluid in and out of adhesive contact. Using a surface force balance we apply electric fields normal to a crossed-cylinder contact and measure directly the adhesive force and surface separation with sub-molecular resolution. Taking advantage of the oscillatory structural force acting between the surfaces across the fluid, which we show to be unaffected by the AC field, we pick between the distinct (quantized) adhesive states through precise tuning of the field. This proof-of-concept indicates exquisite control of surface interactions using an external field.
\end{abstract}

Keywords: adhesion, electric fields, surface forces, ionic liquid

S Supplementary material for this article is available online

(Some figures may appear in colour only in the online journal)

The control of friction and adhesion have been central to technological advancement since ancient times, from starting fires [1] to building monuments to kings [2]. Tribology, the science of adhesion and friction, has progressively adjusted focus over the past century from macro- and mesoscales down to the nanoscale [3-5]. A basic tenet of tribology is that the adhesion and friction between two materials is fully determined by the properties of the surface material and of any added lubricant. Recently, a series of pioneering experiments challenged this view: friction and adhesion may be controlled by means of external stimuli, without need for altering the material

* Author to whom any correspondence should be addressed.

(c) (i) Original content from this work may be used under the terms of the Creative Commons Attribution 4.0 licence. Any further distribution of this work must maintain attribution to the author(s) and the title of the work, journal citation and DOI. components [6, 7]. Strategies for such external control include mechanical excitations $[8,9]$ and photo-switching of friction and adhesion at materials coated in light-responsive molecules [10].

An alternative route to external control of surface interactions is through electrical polarisation of one or both surfaces. An attractive feature of using electrical signals is the ability to switch surface interactions (such as adhesion) 'on' and 'off', remotely and reversibly. Furthermore, electric fields and electrical polarisation can be applied noise-free, require no bespoke chemistry or additional mechanical components, and they can be focussed at particular sites of interest. A few cases of friction and adhesion control with electric fields have been reported [7]. For example, friction has been increased across semiconducting contacts by applying a bias that alters the doping levels locally and thus changes the friction force [11-13]. 
Electric fields have been used to control the friction between two surfaces coated with polyelectrolyte brushes, as the field controls the degree of interpenetration of the brushes in contact [14]. Constant voltages have been used to alter the friction between AFM tips and gold or graphite surfaces separated by thin layers of ionic liquid; applying a potential to the sliding surfaces changes the composition of the boundary layer and thus changes the friction response $[15,16]$. Another example of constant voltage friction control has recently been demonstrated in the surface force balance (SFB), with a gold and mica surface immersed in an aqueous electrolyte. The gold potential can be used to determine if ions will be introduced between the surfaces when they are in contact, and thus produce a different friction response [17].

Here, we demonstrate electric-field control of adhesion using a different mechanism. By applying an alternating (AC) electric field perpendicular to two surfaces with a liquid film between, we introduce a force which can be used to drive the surfaces in and out of adhesive contact. Taking advantage of the oscillatory interaction potential between the surfaces across the liquid, the AC actuation allows us to choose between the available adhesive states and thus provides exquisite control of the adhesion strength. Our measurements, carried out with a SFB, have resolution in surface separation of $\sim 0.1 \mathrm{~nm}$ and therefore provide insight into the adhesion process at the level of individual liquid layers between the surfaces.

The SFB used for these experiments is optimised for measuring the interaction forces between surfaces across fluids as a function of their separation distance with sub-molecular resolution. For the present experiments the SFB was adapted for applying electric fields precisely perpendicular to the confinement direction; the set-up is shown schematically in figure 1, and has been presented recently [18].

For our proof-of-concept experiments we chose an ionic liquid as the fluid medium due to the very strong oscillatory surface forces in ionic liquids compared to the equivalent structural forces in non-charged systems [19-23]. Furthermore, ionic liquids are well known to perform favourably in engineering contexts due to their negligible vapour pressure, chemical stability, resistance to degradation, and lubrication qualities [24, 25]. We apply AC electric fields rather than DC fields because DC fields typically lead to electrochemical degradation and are subject to electrostatic screening in ion-containing fluids. AC fields, on the other hand, act in an unscreened manner if the frequency is higher than the double-layer charging time of the system [26].

The fluid film is confined between the two silver electrodes with mica sheets acting as dielectric spacers coating each electrode. The mica sheets are atomically smooth and precisely uniform in thickness, $T_{\mathrm{m}}$, across the whole area of the two electrodes (figure 1). The mica-coated electrodes are supported on optical lenses with cylindrical curvature $(R \approx 1 \mathrm{~cm})$, mounted in crossed-cylinder geometry, to provide a reproducible point contact. The electrodes are formed by thermal evaporation of Ag to a thickness of $40 \mathrm{~nm}$, chosen so that they can simultaneously act as semi-transparent mirrors for white light interferometry. Collimated white light (from an Ar-Xe arc lamp bulb, Osram) is incident normal to the crossed-cylinders, and the

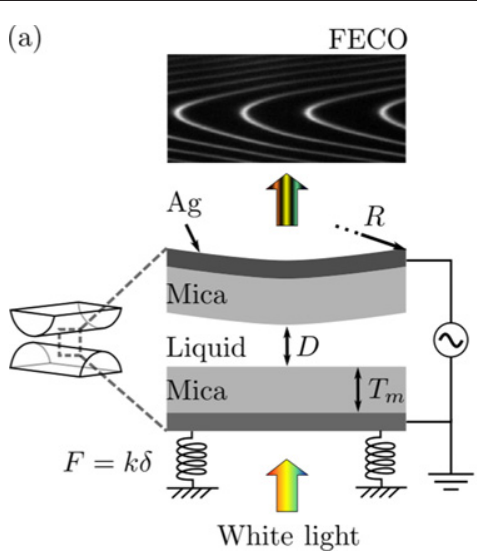

(b)

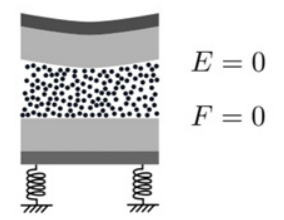

(c)

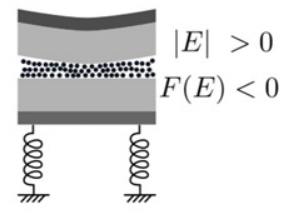

Figure 1. (a) SFB experimental setup, showing the crossed-cylinder geometry and zoom-in to the contact region. The light path is directly normal to the silver electrodes, which act simultaneously as mirrors for interferometric determination of the minimum liquid film thickness, $D$, with sub-molecular resolution. Mica sheets provide a dielectric coating for the electrodes and are of precisely uniform thickness across the whole contact (no steps in the crystal plane). Electric fields are applied normal to the liquid film by way of a time-varying voltage applied to the top electrode while the bottom electrode is held at ground. Forces are measured via the deflection of springs under the bottom electrode. (b) and (c) Schematics showing the effect of applied electric field. The field imposes a normal force which leads to confinement of a controlled number of molecular layers between the surfaces and thus introduces an adhesion determined by the number of liquid layers.

emerging pattern of fringes of equal chromatic order (FECO) is used to determine the closest separation of the surfaces, $D$, within $0.1 \mathrm{~nm}$ [27]. Forces between the surfaces are also determined from the FECO by the deflection of a leaf spring of known spring constant $k$. Electric fields are applied perpendicular to the liquid film by setting up a potential difference between the silver mirror-electrodes, in a manner similar to that first reported by Drummond [14]. Wire attachments are made with a conductive glue (EPON 1004, shell chemicals, mixed with powdered graphite), and bias is applied to the top lens using a signal generator (Keysight 33511B) while the bottom lens is connected to ground. The applied voltage signal is sinusoidal, with root-mean-square amplitude $V_{0}$ and frequency $\nu$. Typical frequencies used in our experiments are 1 to $10 \mathrm{kHz}$, which should be faster than the double layer charging time of our system [18].

Calibration of the FECO in mica-mica direct contact is performed in air in order to determine $T_{\mathrm{m}}$, this later allows for absolute measurement of $D$. An important test carried out at the start of each experiment is the measurement of the capacitor force between the electrodes when an AC field is applied across dry air with the surfaces out of contact. Under these conditions the force can be calculated precisely for our experimental geometry [18], and matching calculation to experiment confirms the quality of all electrodes and connections prior to liquid injection. After calibrations, the ionic liquid (1-butyl1-methylpyrrolidinium bis(trifluoromethanesulfonyl)imide, $\left[\mathrm{C}_{4} \mathrm{C}_{1}\right.$ Pyrr][TFSI], Iolitec $99.5 \%$ ) was injected between the two electrodes.

We first investigated the interaction potential between the surfaces, as a function of $D$, both with and without the applied 


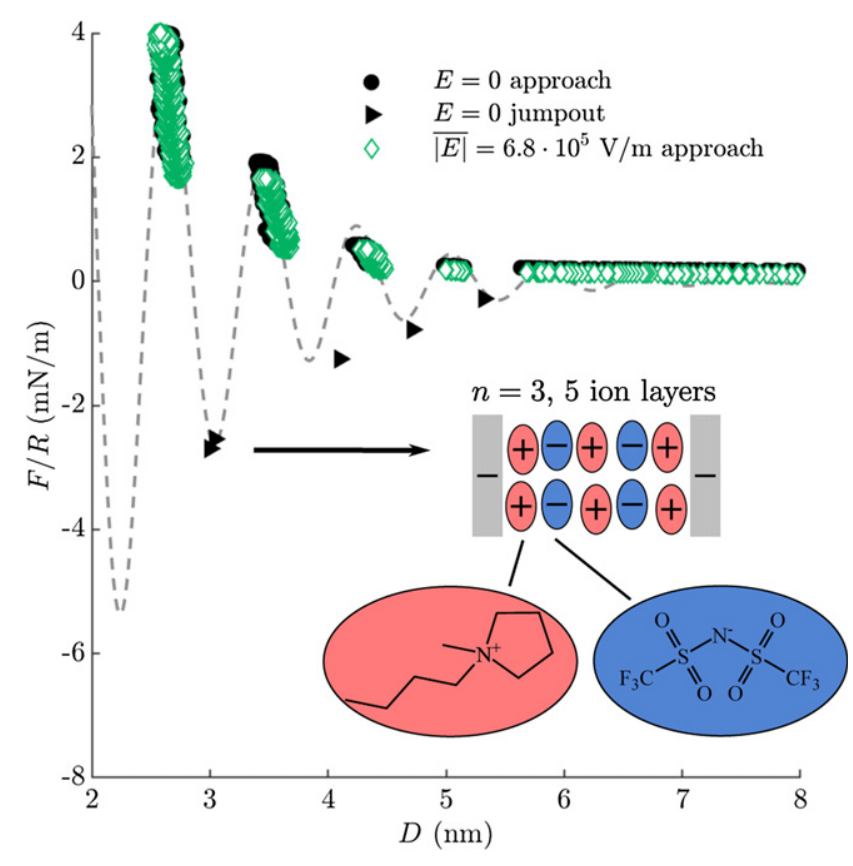

Figure 2. Measured interaction force $F$ between the surfaces (normalised by the radius of curvature $R$ ) as a function of separation distance, $D$, without electric field (black circles and triangles) and with an applied field of amplitude of $5 V_{\text {RMS }}$ and a frequency of $10^{4} \mathrm{~Hz}$ (green diamonds). Note that the steady state force contribution arising from the application of the electric field has already been subtracted from the interaction force in the presence of the electric field. $F / R$ is proportional to the interaction energy per unit area between parallel plates at separation $D$, and so is quantitatively comparable between different contact spots and different experiments. The step-wise increases in $F / R$ measured on approach (black circles, green diamonds) corresponds to sequential squeezing out of layers of molecules from the film. For ionic fluids, the repeat unit involves cation-anion (bi-)layers to maintain electroneutrality [21], so that the third minimum, $n=3$, involves five ion layers as shown in the inset schematic. Data for two deepest minima, $n=1$ (one ion layer) or $n=2$ (three ion layers), are not shown in this experiment. The black triangles correspond to points measured from the jump out of the surfaces from adhesive minima. The dashed line shows a damped oscillatory function of period $\lambda_{i}=0.8 \mathrm{~nm}$ and decay length $\lambda_{o}=1.1 \mathrm{~nm}$ for comparison. The chemical structure of the ionic liquid, 1-butyl-1methylpyrrolidinium bis(trifluoromethylsulfonyl)imide, is also shown.

AC electric field; figure 2. To measure the interaction potential in the absence of an electric field, the surfaces are approached using a mechanical or piezoelectric drive from $D>100 \mathrm{~nm}$, and we record the normal force $F$ from the spring deflection, as a function of the surface separation $D$. To make a measurement of the force profile with the electric field, we initially apply the electric field from a separation of several hundred nanometers; at the point of application of the AC electric field, the surfaces approach each other as described previously [18]. We take care to apply the field at a distance sufficiently large that the surfaces will approach but remain separated by more than $100 \mathrm{~nm}$ when steady state is reached. Then, keeping the electric field on, we approach the surfaces using a mechanical or piezoelectric drive to measure the force profile as would be done without the field. Figure 2 shows the normalised force acting between the surfaces, with applied electric field (green diamonds) and without applied electric field (black circles and triangles). The steady state force from the electric field has been subtracted in the green diamond data. A more detailed explanation of the data acquisition process for the force profiles, with detailed trajectories, is included in the supplemental material (figure S1 (https://stacks.iop.org/JPCM/33/31LT02/mmedia)). Another force profile comparison with and without field, with larger magnitude of the applied field, is shown in supplemental material figure $\mathrm{S} 2$.

The force profile with no electric field applied shows clear discontinuities in distance as the surfaces approach, with repulsive walls of increasing force separated by jumps to closer separations. Retraction of the surfaces from different points on the approach profile reveal negative (adhesive) force minima with increasing depth for decreasing $D$. These features outline the well-established oscillatory structural force acting between surfaces separated by (ionic or molecular) liquids $[22,28,29]$; the oscillations arise from sequential squeeze-out of layers with the oscillatory wavelength determined by the molecular dimensions. In general, the surface force between symmetrical plates across simple molecular or electrolytic liquid can be described by a damped oscillatory function and a monotonically decaying exponential term [29, 30]:

$$
F_{N} / R=A \mathrm{e}^{-D / \lambda_{o}} \cos \left(\frac{2 \pi}{\lambda_{i}} D+\phi\right)+B \mathrm{e}^{-D / \lambda_{\mathrm{s}}}
$$

where the first term on the right represents the oscillatory structural force and the second term arises from electrostatic interaction. In general, the prefactors $A, B$ depend on the surfaces (their charge, polarity, and interaction with the fluid) whereas the decay parameters $\lambda_{o}$ and $\lambda_{\mathrm{s}}$ and wavelength $\lambda_{i}$ are properties of the fluid [31]. $\lambda_{o}$ and $\lambda_{i}$ are determined by the molecular sizes and structural correlations. $\lambda_{\mathrm{s}}$ is an electrostatic screening length; for dilute electrolyte $\lambda_{\mathrm{s}}$ is the Debye screening length, $\lambda_{\mathrm{D}}$ and in ionic liquids $\lambda_{\mathrm{s}}>\lambda_{\mathrm{D}}[32,33]$. For uncharged systems $B=0$; for identical charged plates in electrolytes $B>0$ although usually $A \gg B$. In the present measurements we concern ourselves only with the structural forces. For the ionic liquid studied here, we find that $\lambda_{o}=1.1 \mathrm{~nm}$ and $\lambda_{i}=0.8 \mathrm{~nm}$; these values match the dimensions measured in the past for the same ionic liquid $[34,35]$.

Notably, when comparing the force profiles with and without applied electric field-black circles and triangles vs green diamonds in figure 2-we observe very similar features. The structural force appears independent of the electric field, within our experimental resolution, across all parameters tested, including with different ionic liquids (see SI), different $\mathrm{AC}$ frequencies $\left(\nu \in\left\{10^{3} \mathrm{~Hz}, 10^{4} \mathrm{~Hz}\right\}\right)$, and at different applied voltage $\left(V_{\mathrm{RMS}} \in\{5 \mathrm{~V}, 7 \mathrm{~V}\}\right)$.

With the oscillatory force-law between the surfaces thus established, we next explore the possibility of surface actuation by switching the AC field. Figure 3 summarises the results of an example experiment. The surfaces were initially placed at a separation $D=D_{0}$ (using a mechanical drive), and with no applied field. We varied $D_{0}$ in the range $30 \mathrm{~nm}<D_{0}<400 \mathrm{~nm}$. An AC field was then applied (frequency $\nu$, RMS magnitude $V_{0}$ starting at time $t_{\mathrm{on}}$ ). The effect of the field was to drive 


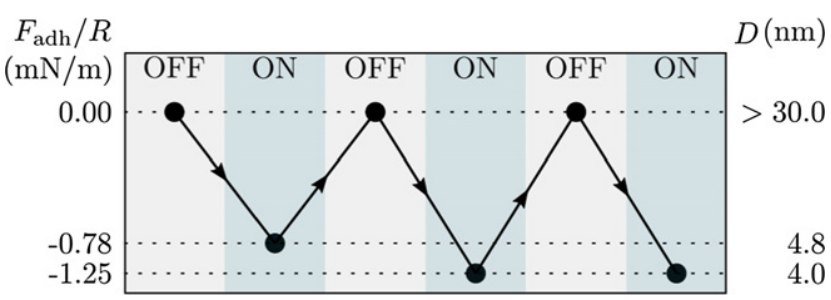

Figure 3. Cycling the surfaces in and out of adhesive contact using the electric field. Initially $E=0$ and $D>30 \mathrm{~nm}$, therefore no surface force is measured. An AC field is then applied ( $V_{0}=7 V_{\mathrm{RMS}}, \nu=10 \mathrm{kHz}$ ) which drives the surfaces to a separation $D=4.8 \mathrm{~nm}$, corresponding to the fifth minimum $n=5$ (with nine ion layers) and an adhesive force of $F_{\text {adh }} / R=$ $-0.78 \mathrm{mN} \mathrm{m}^{-1}$. When the field is turned off, the relaxation effect exerts enough external force to pull the surfaces out of the adhesive minimum to a separation $D>30 \mathrm{~nm}$, and the surfaces are free (no surface force is measured). Switching the field on again allows the surfaces to be brought back into adhesive contact; this time by applying a greater AC field-induced force the surfaces reach a minimum at $D=4.0 \mathrm{~nm}(n=4$; for which there are seven ion layers and $F_{\text {adh }} / R=-1.25 \mathrm{mN} \mathrm{m}^{-1}$ ). The effect is reversed again when the field is switched off, and then re-applied at the same magnitude to reproducibly reach the same minimum at $D=4.0 \mathrm{~nm}$. For this experiment $k=140.6 \mathrm{~N} \mathrm{~m}^{-1}, T_{\mathrm{m}}=3.3 \mu \mathrm{m}$, and $R=$ $9.6 \mathrm{~mm}$. Further examples are shown in the supplemental material.

the surfaces together [18], and as the separation decreased to $\leqslant 6 \mathrm{~nm}$, the field was sufficient to overcome maxima in the oscillatory forces and so drive the surfaces between local energy minima. An example $D(t)$ curve during application of the AC field is shown in figure S3 in the supplement. As shown in the summary example, figure 3 , we are able to choose $D_{0}$, $V_{0}$, and $t_{\text {off }}$ such that we control the adhesive minimum reached under the AC field. The effect is reversible, with the surfaces jumping out of adhesive contact when the field is switched off. Repeated cycles, switching the field on and off, allows for switching in and out of adhesive minima.

The force between the surfaces brought about by applying an AC field across fluid depends on (i) magnitude of the field, with the 'steady state' force imposed by the field, $F^{\text {ss }}$, scaling as $F^{\mathrm{ss}} \sim V_{0}^{2}$ (for both molecular and ion-containing fluid media) $[18,36]$, (ii) dielectric and electrokinetic properties of the medium $[18,36]$, and (iii) geometric factors $D_{0}$, $T_{\mathrm{m}}$, and $R$. For dielectric fluids, such as molecular liquids, the force between the electrodes is simply the attraction between charged capacitor plates and has been established quantitatively in our experimental setup [18]. When the fluid contains ions-either as a pure ionic liquid as in the present work or as a diluted electrolyte-the magnitude of $F^{\mathrm{ss}}$ is substantially larger than this simple capacitor force and evolves over slower timescales than the viscous drainage timescale, as reported recently $[18,36]$. The origin of this additional force induced by the action of the electric field on the mobile ions remains intriguing and is not yet fully resolved, although strong clues of the importance of ion asymmetry have been provided by Richter and co-workers [36]. The effect is reminiscent of the observed colloidal forces under AC fields in electrolyte reported by Woehl et al [37, 38]; in their later calculations [39] it was pointed out that oscillating electric fields in electrolytes can create a long-range steady field. Separately, Stone, Holyst and Drummond have suggested the origin of the additional force lies in an excess osmotic pressure due to ions drawn into the region of high field from surrounding reservoirs [36]. Here, without attempting to resolve this mechanistic question, we simply make use of the property that $F^{\mathrm{ss}}=K_{\text {liq }} V_{0}^{2}$, with fitting parameter $K_{\text {liq }}$ dependent on the liquid and contact geometry.

The relationship between applied voltage and the accessible adhesive minima is inspected in more detail for one example experiment in figure 4 . Smaller voltages allow switching in and out of only the shallower adhesive minima, while larger voltages can be applied to access the deeper minima. Since the minima in the interaction potential of equation (1), are at $\left(\frac{2 \pi}{\lambda_{i}} D_{\min }+\phi\right)=(2 n-1) \pi(n \in 1,2, \ldots)$, giving $D_{\min }=$ $n \lambda_{i}+\left(\frac{\lambda_{i}}{2}-\frac{\lambda_{i} \phi}{2 \pi}\right)$ and $F_{\text {adh }} / R=-A \mathrm{e}^{-D_{\min } / \lambda_{o}}$, we have that $\ln \left|F_{\text {adh }}\right|=c-n \frac{\lambda_{i}}{\lambda_{o}}$ with $c$ constant. The semi-log plot in figure 4(a) shows the measured $\ln \left|F_{\text {adh }}\right|$ vs $D$ (black points; fitted dashed line). Since switching is possible when $\left|F^{\mathrm{ss}}\right|>$ $\left|F_{\text {adh }}\right|$, plotting points in $V_{0}^{2}$ vs film thickness where adhesion switching occurs or otherwise, figure 4(a), creates a phase diagram separating the switching and no-switching regions in $D$, $V_{0}$. The fact that the line $\ln \left|F_{\text {adh }}\right|=c-n \frac{\lambda_{i}}{\lambda_{o}}$ must define the 'switching boundary' allows us to determine the parameter $K_{\text {liq }}=1.38 \mathrm{~N} \mathrm{~V}^{-2}$. Figure S2(b) shows detail and description of individual measurements. Figure 4(b) shows schematically how the applied force arising from an AC field allows for adhesion in any minimum in the oscillatory profile for which $\left|F^{\mathrm{ss}}\right|>\left|F_{\text {adh }}\right|$. Since $F^{\mathrm{ss}}$ scales with $V_{0}^{2}$, small changes to $V_{0}$ allow for sensitive tuning of the maximal force applied by the $\mathrm{AC}$ field and so controlled adhesion in deeper energy minima.

In considering the origin of the adhesion control, it is worth interrogating the (perhaps counterintuitive) observation that the normal oscillatory potential, figure 2 , is not altered by application of an AC field and yet the surface force introduced by switching on the field is sufficient to drive the surfaces in a substantial and controlled way. This useful phenomenon arises because of the different range of forces relevant in determining the behaviours: the oscillatory structural force arises from strong solid-liquid surface forces of decay length $\lambda_{o} \sim 1 \mathrm{~nm}$ [35], while the AC field exerts a body force acting throughout the media between the electrodes [18]. When the crossedcylinders approach to small $D$ the interaction potential is dominated by the strong oscillatory surface forces at the point asperity determined by the liquid; whereas applied AC field acts across the whole region between the electrodes and so the resulting force is dominated by action away from the asperity contact. In this way, the field can be used as an external drive to explore the energy landscape imposed (determined) by the liquid between the surfaces. In principle, such an external drive could be provided by other mechanisms, such as an electromagnet or piezoelectric actuator; our present measurements present one such mechanism with particularly fine control.

The timescales for the action of the AC electric field to reach steady state in this proof-of-concept experiment are of the order of $10^{2}-10^{3} \mathrm{~s}$. These timescales may be slow for some practical applications, but it may be possible to optimise this response time by varying factors such as the geometry of 
(a)

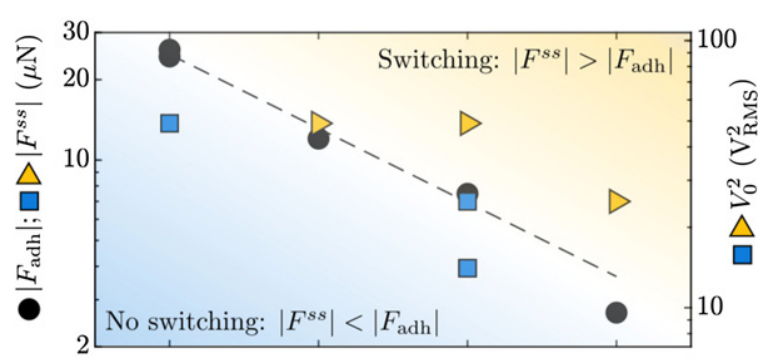

(b)

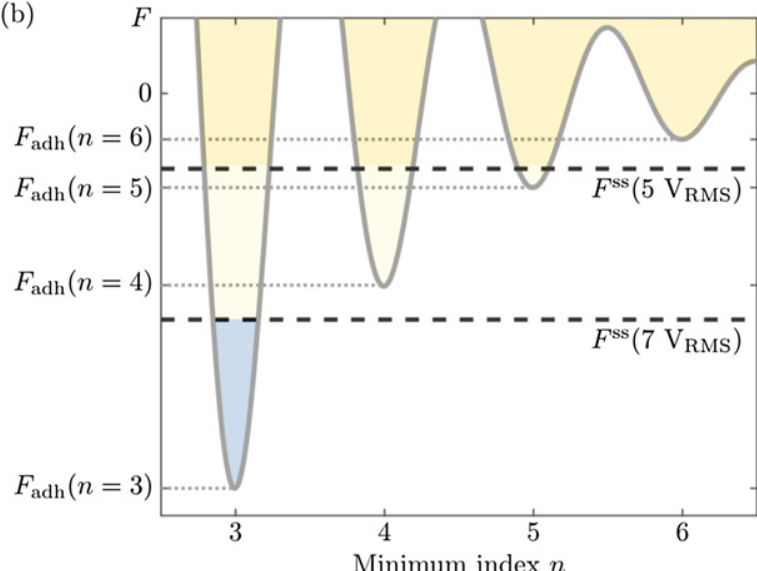

Figure 4. Tuning the voltage allows for adhesion and de-adhesion in different energy minima. (a) Summary phase diagram showing, for one example experiment, the regions in voltage (right axis;

logarithmic scale) and film thickness (indicated by minimum index $n$, bottom axis) where the AC field was observed to actuate surfaces in and out of adhesive minima (yellow triangles and yellow shaded region), and where the AC field was insufficient to switch in and out of the adhesive minima (blue squares and blue shaded region). Black circles show the magnitude of the adhesive minima (left axis; logarithmic), and the line of these minima divides the switching region where $\left|F^{\mathrm{ss}}\right|>\left|F_{\text {adh }}\right|\left(\right.$ with $F^{\mathrm{ss}}=K_{\mathrm{liq}} V_{0}^{2}$ ) and the no-switching region where $\left|F^{\mathrm{ss}}\right|<\left|F_{\text {adh }}\right|$. For these experiments, $V_{0}=3.75,5$ and $7 V_{\mathrm{RMS}}, T_{\mathrm{m}}=3.3 \mu \mathrm{m}$ and $\nu=10^{4} \mathrm{~Hz}$.

(b) Diagram showing the intersection of an oscillatory force profile, for the $n=3,4,5$, and 6 minima, and the maximal force applied by switching on/off an AC field of two different magnitudes. Applying an AC field can exert a maximal force of $F^{\text {ss }}$ which allows for adhesion and de-adhesion into any force minimum of smaller magnitude.

the experiment. Some additional preliminary measurements indicate that the timescale for response is dependent on the spot radius of curvature, $R$, with faster, stronger responses for smaller radii of curvature. It is possible that the AC field effect in systems with ionic liquid can be accelerated by optimising the geometry.

In summary, we demonstrate exquisite control over surface adhesion using an externally applied electric field. The unscreened electric field exerts a body force acting over long range and with magnitude scaling with the square of the applied potential difference, allowing for actuation of the surfaces in a highly controlled way. With the gap between the surfaces filled by an ionic liquid, the surface forces over nanometric distances are oscillatory; each minimum in the oscillatory potential provides an adhesive well of differing depth. We show that tuning the field to apply forces matching a desired adhesive minimum can be used to choose between the different adhesive states. Looking ahead, the facility to switch between adhesive minima opens the possibility of switching between quantized friction states. It is well established that the friction acting between surfaces lubricated by thin ionic films follows multiple discrete friction-load laws, each with different friction coefficients, determined by the number of liquid layers in the film [34]. Introducing the prospect of switching between adhesive minima using an external field opens the way to switching between these distinct friction states in a reversible way. Such principles could be applied for controlled release of particles, electro-actuation, and breaking at the micro- and nanoscale.

\section{Acknowledgments}

CSP-M is grateful for support from a UKRI Future Leaders Fellowship (MR/S032312/1), and for a Junior Research Fellowship from Trinity College, Oxford. SP, TG and CSP-M acknowledge funding from the European Research Council (under Starting Grant No. 676861, LIQUISWITCH) and The Leverhulme Trust (RPG-2015-328). We thank Dr. Alexander Smith for some data used in the supplemental figures.

\section{Data availability statement}

The data that support the findings of this study are available upon reasonable request from the authors.

\section{ORCID iDs}

Carla S Perez-Martinez (D) https://orcid.org/0000-0002-55740319

Timothy S Groves (D) https://orcid.org/0000-0003-2168-2437 Susan Perkin (D) https://orcid.org/0000-0002-5875-5217

\section{References}

[1] Gowlett J A J 2016 Phil. Trans. R. Soc. B 37120150164

[2] Fall A, Weber B, Pakpour M, Lenoir N, Shahidzadeh N, Fiscina J, Wagner C and Bonn D 2014 Phys. Rev. Lett. 112 3-6

[3] Bowden F and Tabor D 1950 The Friction and Lubrication of Solids (Oxford: Clarendon)

[4] Mo Y, Turner K T and Szlufarska I 2009 Nature 457 1116-9

[5] Urbakh M and Meyer E 2010 Nat. Mater. 9 8-10

[6] Carpick R W 2006 Science 313 184-5

[7] Krim J 2019 Front. Mech. Eng. 5 1-22

[8] Socoliuc A, Gnecco E, Maier S, Pfeiffer O, Baratoff A, Bennewitz R and Meyer E 2006 Science 313 207-10

[9] Capozza R, Vanossi A, Vezzani A and Zapperi S 2009 Phys. Rev. Lett. 103085502

[10] Blass J, Bozna B L, Albrecht M, Krings J A, Ravoo B J, Wenz G and Bennewitz R 2015 Chem. Commun. 51 1830-3

[11] Park J Y, Ogletree D F, Thiel P A and Salmeron M 2006 Science 313186

[12] Park J Y, Qi Y, Ogletree D F, Thiel P A and Salmeron M 2007 Phys. Rev. B 76064180

[13] Qi Y, Park J Y, Hendriksen B L, Ogletree D F and Salmeron M 2008 Phys. Rev. B 77184105

[14] Drummond C 2012 Phys. Rev. Lett. 109154302

[15] Sweeney J, Hausen F, Hayes R, Webber G B, Endres F, Rutland M W, Bennewitz R and Atkin R 2012 Phys. Rev. Lett. 109155502 
[16] Li H, Wood R J, Rutland M W and Atkin R 2014 Chem. Commun. 50 4368-70

[17] Tivony R, Zhang Y and Klein J 2021 J. Phys. Chem. C 125 3616-22

[18] Perez-Martinez C S and Perkin S 2019 Soft Matter 15 4255-65

[19] Atkin R and Warr G G 2007 J. Phys. Chem. C 111 5162-8

[20] Bou-Malham I and Bureau L 2010 Soft Matter 6 4062-5

[21] Perkin S, Albrecht T and Klein J 2010 Phys. Chem. Chem. Phys. 12 1243-7

[22] Horn R G, Evans D F and Ninham B W 1988 J. Phys. Chem. 92 3531-7

[23] Ueno K, Kasuya M, Watanabe M, Mizukami M and Kurihara K 2010 Phys. Chem. Chem. Phys. 12 4066-71

[24] Fedorov M V and Kornyshev A A 2014 Chem. Rev. 114 2978-3036

[25] Lhermerout R, Diederichs C and Perkin S 2018 Lubricants 69

[26] Bazant M Z, Thornton K and Ajdari A 2004 Phys. Rev. E 70 021506

[27] Israelachvili J N 1973 J. Colloid Interface Sci. 44 259-72

[28] Horn R G and Israelachvili J N 1981 J. Chem. Phys. 75 1400-11

[29] Ludwig M and von Klitzing R 2020 Curr. Opin. Colloid Interface Sci. 47 137-52
[30] Coles S W, Smith A M, Fedorov M V, Hausen F and Perkin S 2018 Faraday Discuss. 206 427-42

[31] Evans R, Henderson J R, Hoyle D C, Parry A O and Sabeur Z A 1993 Mol. Phys. 80 755-75

[32] Smith A M, Lee A A and Perkin S 2016 J. Phys. Chem. Lett. 7 2157-63

[33] Coles S W, Park C, Nikam R, Kanduc M, Dzubiella J and Rotenberg B 2020 J. Phys. Chem. B 124 1778-86

[34] Smith A M, Lovelock K R J, Gosvami N N, Welton T and Perkin S 2013 Phys. Chem. Chem. Phys. 15 15317-20

[35] Smith A M, Lee A A and Perkin S 2017 Phys. Rev. Lett. 118 096002

[36] Richter L, Zuk P J, Szymczak P, Paczesny J, Bak K M, Szymborski T, Garstecki P, Stone H A and Drummond C 2020 Phys. Rev. Lett. 125056001

[37] Woehl T J, Chen B J, Heatley K L, Talken N H, Bukosky S C, Dutcher C S and Ristenpart W D 2015 Phys. Rev. X 5 011023

[38] Bukosky S C and Ristenpart W D 2015 Langmuir 31 9742-7

[39] Hashemi Amrei S M H, Bukosky S C, Rader S P, Ristenpart W D and Miller G H 2018 Phys. Rev. Lett. 121185504 